\title{
Electronic structure of hydrogen in $\mathrm{Al}$
}

\author{
N SINGH and J SINGH' \\ Department of Physics. M D University, Roblak 12400), India \\ 'Department of Physics, Punjah Agricultural University, Ludhiana 141004 , India
}

\begin{abstract}
bstract. The impurity induced charge density of proton in Al is calculated by solving the Sichrödinger equation self-consistently. The lattice contribution and the lattice dilation are included through spherical solid model potential and Blatt correction. respectively. The proton is kept at an octahedral site. The resulting phase shifts have been used to estimate the residual resistivity for dilute $\mathrm{Al} \cdot \mathrm{H}$ system. This model does not favour the formation of $\mathrm{AiH}$, 1.c., hydrogen exists in Al matrix as a free ion.
\end{abstract}

Keywords. Spherical solid model potential; induced charge density; residual resistivity; impurity potential.

\section{Introduction}

When hydrogen atoms iffuse in a meral, they dissociate into protons and electrons. Protons being positively charged are screened by conduction electrons. They displace the host ions from the equilibrium positions. As a result, the positive charge density in the vicinity of the proton changes.

The first linear theory of proton in $\mathrm{Cu}$ was proposed by Friedel (1952). It is now well established that the screening of the proton is nonlinear. Popovic et al (1976) have calculated the electronic structure, activation energy and heat of solution of hydrogen in simple metals using the density functional theory (Kohn and Sham 1965). Almbladh and von Barth (1976), Kahn et al (1980) and Perrot and Rasolt (1981) have extended the study of Popovic et al (1976) by including the lattice contribution through a simpie form of spherical solid model potential.

Jena and Singwi (1978) added the first gradient correction of exchange-correlation potential and found s-type bound state of the proton. Manninen and Nieminen (1979) added the lattice contribution in a more comprehensive way through the spherical solid model potential and calculated the heat of solution of hydrogen muonium in simple metals.

In some of the above studies, size effect is neglected. In others, both size effect and lattice contributions have been neglected. These contributions change considerably the electronic structure, effective potential and self-energy and residual resistivity of $\mathrm{H}^{+}$in Al by including the contribution of size effect and discrete lattice. This model does not favour the formation of $\mathrm{AlH}$.

\section{Theory}

\subsection{Size effect}

The hydrogen impunty at an interstitial position displaces the host ions away from the equilibrium position. Therefore, the positive charge density decreases and the electron charge density increases in the vicinity of the impurity. As a result of the dilation, the net 
charge of the proton is given as (Beal-Monod and Kohn 1968; Flynn and Stoneham 1970):

$$
Z=Z_{\mathrm{i}}-(\delta V / V) Z_{\mathrm{H}}
$$

where $Z_{\mathrm{i}}$ and $Z_{\mathrm{H}}$ are the valence of impurity and host atom, respectively. In the continuum model of the lattice, the fractional charge in the host atom volume $\delta V / V \mathrm{can}$ be calculated as has been suggested by Blatt (1957).

\subsection{Spherical solid model potential}

The spherical solid model potential is defined as the sum of spherical average of the host-ion potential and electrostatic potential of the positive background of jellium (Manninen and Nieminen 1979):

$$
V_{\mathrm{ss}}(\mathbf{r})=-(1 / 4 \pi) \int \mathrm{d} \cap \sum_{l} w\left(\mathbf{r}-\mathbf{r}_{1}\right)+\int \mathrm{d} \mathbf{r}^{\prime} n_{0} /\left|\mathbf{r}-\mathbf{r}^{\prime}\right|,
$$

Here $\mathrm{d} \cap$ is the elementary solid angle. $n_{0}\left(=3 / 4 \pi r_{\mathrm{s}}^{3}\right)$ is the uniform charge density of the host and $r_{\mathrm{s}}$ the electron density parameter. For simplicity we consider the Ashcroft model potential for bare ion $w(r)$, i.e.

$$
w(r)=\left(Z_{\mathbf{H}} / r\right) \theta\left(r-r_{\mathbf{c}}\right)
$$

where $r_{\mathrm{c}}$ is the potential parameter and $\theta(r)$ the step function.

\subsection{Non linear screening}

Single electron eigen values $\varepsilon_{k}$ and corresponding radial wave function $R_{l k}(r)$ are obtained by solving the following one particle radial equation:

$$
\left[-\frac{1}{2} \mathrm{~d}^{2} / \mathrm{d} r^{2}+V_{\text {eff }}(\mathbf{r})+l(l+1) / 2 r^{2}-\varepsilon_{k}\right] r R_{l k}(\mathbf{r})=0 .
$$

For the continuum states $\varepsilon_{k}=k^{2}$, zero energy is taken to be the potential far from the impurity where its effect almost vanishes. Thus the effective potential field, in which the electron moves, is

$$
V_{\mathrm{eff}}(\mathbf{r})=\Phi(\mathbf{r})+V_{\mathrm{ss}}(\mathbf{r})+V_{\mathrm{xc}}(n(\mathbf{r}))
$$

The parametrized form of $V_{\mathrm{xc}}(n(\mathbf{r}))$ proposed by Gunnarson and Lundquist (1976) has been adopted for the detailed calculations. Following Popovic et al (1976), the induced electron charge density is written as

$$
\begin{aligned}
\delta n_{\mathrm{i}}(r) & =n(r)-n_{0}(r), \\
& =1 / \pi^{2} \int_{0}^{K_{\mathrm{F}}} \mathrm{d} k k^{2} \sum_{l=0}^{x}(2 l+1)\left[R_{l k}^{2}(r)-J_{l}^{2}(k r)\right]+2 R_{b}^{2}(r),
\end{aligned}
$$

where $k$ is the electron wave vector and $k_{\mathrm{F}}$ the Fermi momentum. The sum over angular momentum quantum number $l$ in (6) converges very rapidly. This is because the 
effective potentual is localized so that the radial wave functions $R_{l k}(r)$ for large / will differ from the unperturbed electron Bessel functions $j_{l}(k r)$. s-type bound state may occur some time, therefore, the band structure charge density is also added in (6). In the present calculation the bound states with two electrons are considered. The electrostatic potential $\phi(\mathbf{r})$ is obtained by solving the Poisson equation

$$
\nabla^{2} \phi(\mathbf{r})=-4 \pi\left(Z_{\mathrm{i}} \delta(\mathbf{r})+n_{0}-n(\mathbf{r})\right),
$$

following the procedure of Manninen et al (1975) for achieving better convergency.

An alternative simple one-parameter screened Coulomb potential function, which has been shown to provide an accurate description of a proton impurity in both electron gas and a transition metal (Sholl and Smith 1978) is

$$
V_{\mathrm{sc}}(r)=-Z(1+\beta r / 2) \exp (-\beta r) / r .
$$

The parameter $\beta$ is chosen so that $V_{\mathrm{sc}}(r)$ satisfies the Friedel sum rule. By solving the Poisson equation we find that $V_{\mathrm{sc}}(r)$ yields a simple analytical form of induced charge density:

$$
\delta n_{\mathrm{i}}(r)=\left(Z \beta^{3} / 8 \pi\right) \exp (-\beta r)
$$

Equation (9) yields a finite value of $\delta n_{\mathrm{l}}(r)$ at $r=0$. The simple function $V_{\mathrm{sc}}(r)$ and $\delta n_{\mathrm{i}}(r)$ given by ( 8 ) and (9), respectively, can be easily used to evaluate the self-energy of the induced charge analytically. As a result, the self-energy, defined as

$$
E_{\mathrm{s}}=\frac{1}{2} \int V_{\mathrm{sc}}(r) \delta n_{\mathrm{i}}(r) \mathrm{d} \mathbf{r},
$$

reduces to

$$
E_{\mathrm{s}}=\cdots 3 L^{2} \beta / 32 \text { a.u. }
$$

However, the charge density corresponding to Thomas-Fermi potential comes out to be $-Z^{2} \beta / 4$ a.u.

\section{Calculations and results}

\subsection{Effective charge on the proton}

The effective charge on the proton in Al metal is estimated using (1). The value of $\delta \mathrm{V} / \mathrm{V}$ and $Z$ are given in table 2 . The proton charge is reduced by $40 \%$ by the Blatt correction (Blatt 1957).

\subsection{Spherical solid model potential}

The potential parameter $r_{\mathrm{c}}$, Madelung constant $\alpha_{\mathrm{M}}$, octahedral coordinates and the atomic radius $r_{0}$ for $\mathrm{Al}$ are given in table 1 . The potential $V_{\mathrm{ss}}(r)$ is terminated at $r=8$ a.u. because it becomes negligibly small for larger values of $r$. The calculated $V_{\mathrm{ss}}(r)$ for $\mathrm{Al}$ is shown in figure 1 . The number of nearest neighbours and their position with respect to 
Table 1. The host parameters used in the calculations. $r_{0}$ is the atomic radius, $Z_{13}$ is the valence, $r_{\mathrm{c}}$ is the Ashcroft model potential parameter and $\alpha_{M}$ is the Madelung constant for octahedral site.

\begin{tabular}{lccccc}
\hline Parameter & $r_{\mathrm{o}}$ (a.u.) & $Z_{11}$ & $r_{\mathrm{c}}$ (a.u.) & $\alpha_{\mathrm{M}}$ & oct. site \\
\hline 2.985 & 3 & $1 \cdot 12$ & 0.42586 & $\left(\frac{1}{2} \frac{1}{2} \frac{1}{2}\right)$ \\
\hline
\end{tabular}

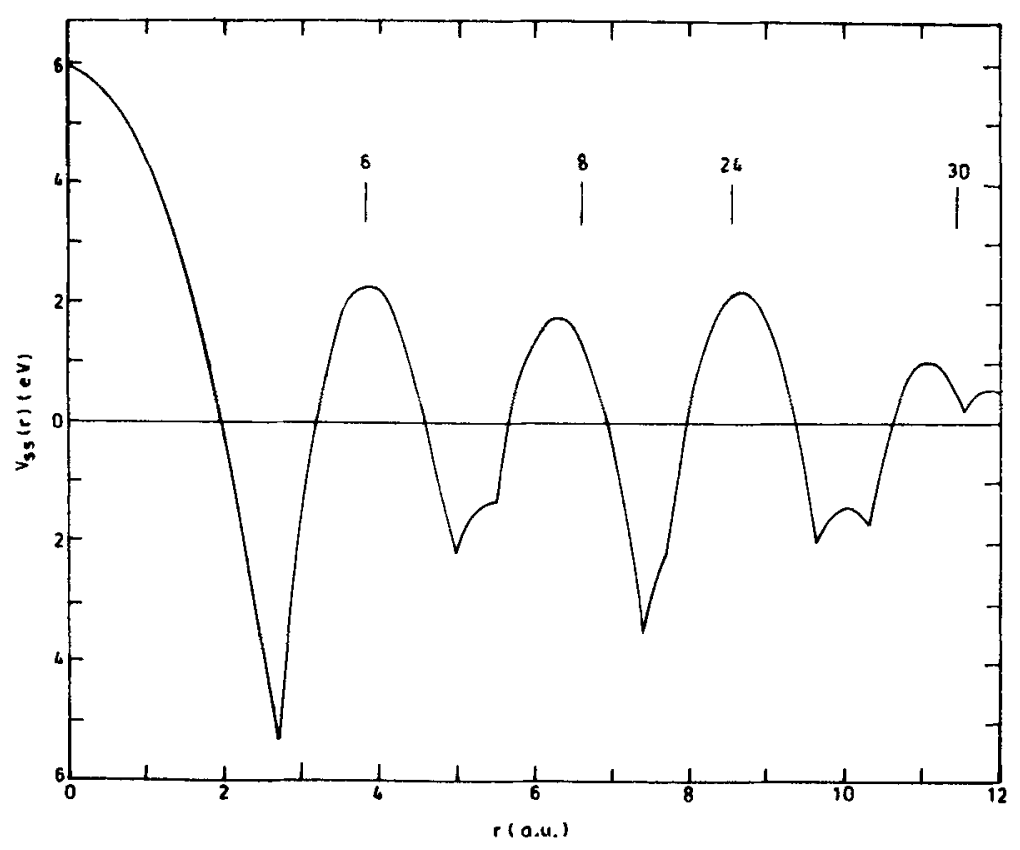

Figure 1. The spherical solid model potential $V_{\mathrm{ss}}(r)$ for $\mathrm{Al}$ around the octahedral site. The position and number of nearest neighbours to the octahedral site are also indicated.

the octahedral site are also indicated in the figure 1. $V_{\mathrm{ss}}(r)$ has maxima around neighbouring ion shells and minima between the two shells. The potential depends upon $Z_{\mathrm{H}}, r_{\mathrm{c}}$ and the octahedral site.

\subsection{Phase shifts and impurity potential}

We have followed the procedure of Manninen et al (1975) for solving (4)-(7), selfconsistently. The s-type bound states are also included. The radial part of the Schrödinger equation is solved numerically in steps of 0.05 a.u. up to a radius of $R_{0}=10$ a.u. using Fox-Goodwin VI method. The phase shifts are calculated by matching the numerical solution with asymptotic solution at $R_{0}$. The self-consistency of the calculation is checked using the Friedel sum rule

$$
Z=2 / \pi \sum_{l=0}^{6}(2 l+1) \delta_{l}\left(k_{\mathrm{F}}\right)
$$

In (12) $l$ is an orbital quantum number and $\delta_{l}\left(k_{\mathrm{F}}\right)$ are scattering phase shifts at the 
Table 2. $\delta l / V$ is the fractional change in atomic volume. $/$ is the offectuve charge on impurify, $\beta$ is potential parameter, $x^{\prime}$ and $\beta^{\prime}$ are potential parameters, $V_{w}^{\prime}(r)$ is spherical solid

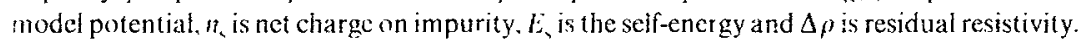

\begin{tabular}{|c|c|c|c|}
\hline \multirow{2}{*}{$\begin{array}{l}\text { Physical quantities } \\
\text { or/V }\end{array}$} & \multirow{2}{*}{$\begin{array}{c}\text { Present model } \\
\left(V_{4}^{\prime}(r) \text { is included }\right) \\
0.13\end{array}$} & \multicolumn{2}{|c|}{$\begin{array}{c}\text { Jcllium model } \\
\left(V_{,}(r) \text { is not included }\right)\end{array}$} \\
\hline & & 0.13 & 0 \\
\hline$\measuredangle$ & 0.61 & 0.61 & $1 \cdot 0$ \\
\hline$\ddot{x}$ & & 1.0222 & $1 \cdot 1520$ \\
\hline$\beta^{\prime}$ & & 1.2693 & $1 \cdot 1709$ \\
\hline$\beta$ & 1.6877 & $1 \cdot 8124$ & 2.6355 \\
\hline$n_{4}(\mathrm{c})$ & 0.43 & 0.41 & 0.57 \\
\hline$E_{\mathrm{c}}($ ryd $)$ & -0.1177 & $-0 \cdot 1264$ & -0.1839 \\
\hline \multicolumn{4}{|l|}{${ }_{1} \mu(\mu \Omega \mathrm{cm}$ at $\%)$} \\
\hline expt. & & & - \\
\hline cal. & 0.2936 & 0.2870 & 0.6847 \\
\hline
\end{tabular}

Table 3. The scattering phase shifts $\delta_{1}\left(k_{1}\right)$ for proton $\left(\mathrm{H}^{+}\right)$in $\mathrm{Al} .1=0,1,2, \ldots$ represents s, p. d..... phase shifts.

\begin{tabular}{lllr}
\hline 1 & Present model & \multicolumn{2}{c}{ Jellium model } \\
\hline 0 & 0.682792 & 0.641037 & 1.086077 \\
1 & 0.129128 & 0.080010 & 0.121071 \\
2 & 0.026103 & 0.012006 & 0.019446 \\
3 & 0.006210 & 0.001979 & 0.003203 \\
4 & 0.001475 & 0.000247 & 0.000372 \\
9 & 0.000621 & 0.000075 & 0.000015 \\
0 & 0.000391 & 0.000012 & -0.000133 \\
\hline
\end{tabular}

Fermi-energy $E_{\mathrm{F}}$. The first seven phase shifts $\delta_{l}\left(k_{\mathrm{F}}\right)$ have been used to satisfy (12) as their values become too small for $I>6$. The induced electron density $\delta n_{\mathrm{i}}(r)$ is calculated by using the first seven partial radial waves obtained by varying $k$ from $0 \rightarrow k_{\mathrm{F}}$. The phase shifts so calculated are given in table 3 . The $s$ and $p$ phase shifts are found to be dominating. We have also calculated the induced charge density in jellium following the procedure of Popovic et al (1976) without including the size effect and then by including it. The phase shifts calculated without $V_{s i}(v)$ converge fast. It is found that the size effect reduces the magnitude of the phase shifts considerably. Therefore, we conclude that both $V_{\mathrm{ss}}(r)$ and size effect change the scattering of impurity at Fermi surface.

The impurity potential so calculated are shown in figure 2 . The potentials are strongly attractive in the vicinity of the impurity and exhibit Friedel oscillations at large distances. A comparison of $\delta V_{\mathrm{i}}(r)$ calculated by including size effect with that calculated by including both size effect and $V_{n}(r)$ show that the lattice contribution enhances the attractive nature of $\delta V_{i}(r)$.

\subsection{Electronic charge density and charge transfer}

The calculated impurity induced charge density $\delta n_{i}(r)$ in $\mathrm{Al}$ is shown in figure 3 . There is pile up of charge on the impurity site. It decreases rapidly as we move away from the 


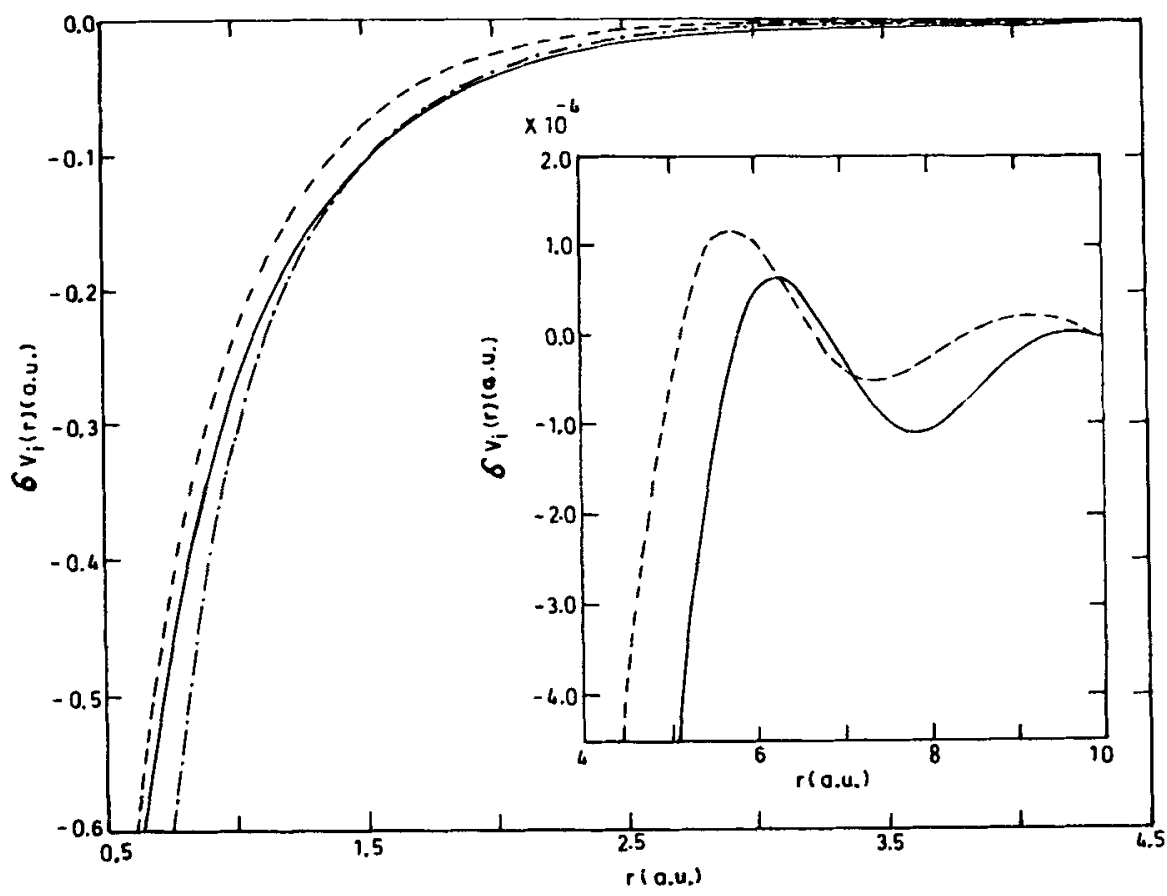

Figure 2. The proton induced self-consistent potential $\delta V_{i}(r)$ in $\mathrm{Al}$. The solid line represents $\delta V_{i}(r)$ by including both $V_{\mathrm{ss}}(r)$ and size effect. Dash and dash-dot lines represent $\delta V_{i}(r)$ with size effect and without size effect, respectively, in jellium model (Popovic et al 1976).

centre and becomes oscillatory at large $r$. As compared to $\delta n_{i}(r)$ in jellium model (Popovic et al 1976) we found that the lattice contribution and size effect both reduce the magnitude of $\delta n_{\mathrm{i}}(r)$.

An $s$-type very shallow bound state of energy $-0.7450 \times 10^{-8} \mathrm{ryd}$. is also found in our calculations. This suggests that there is no formation of $\mathrm{AlH}$, i.e. the protons exist at interstitial sites in Al matrix as free ions. The impurity induced self-energy is calculated using (11). The calculated $E_{\mathrm{s}}$ for proton in $\mathrm{Al}$ is found to be $-0.1177 \mathrm{ryd}$. The electron charge density $\delta n_{\mathrm{i}}(r)$ is integrated in the hydrogen core (1 a.u.) to give $Z_{\text {core }}$, which comes out to be $0 \cdot 18 \mathrm{e}$. This gives the net charge on the proton $n_{\mathrm{s}}=$ $Z-Z_{\text {core }}=0.43 \mathrm{e}$. Comparing with the $n_{\mathrm{s}}=0.41 \mathrm{e}$ in jellium model we found that the lattice effect reduces the net charge on proton due to enhanced scattering.

\subsection{Residual resistivity}

The residual resistivity $\Delta \rho$ for $\mathrm{Al}-\mathrm{H}$ is estimated using the expression for liquid crystals (Blatt 1957)

$$
\Delta \rho=\frac{2 \cdot 732}{k_{\mathrm{F}} Z_{\mathrm{H}}} \sum_{0}^{6}(l+1) \sin ^{2}\left(\delta_{l}-\delta_{l+1}\right)
$$

using the phase shifts given in table 3 . The value of residual resistivity is found to be $0.2936 \mu \Omega \mathrm{cm} / \mathrm{at} . \%$. The corresponding value in jellium is found to be $0 \cdot 2870 \mu \Omega$ 


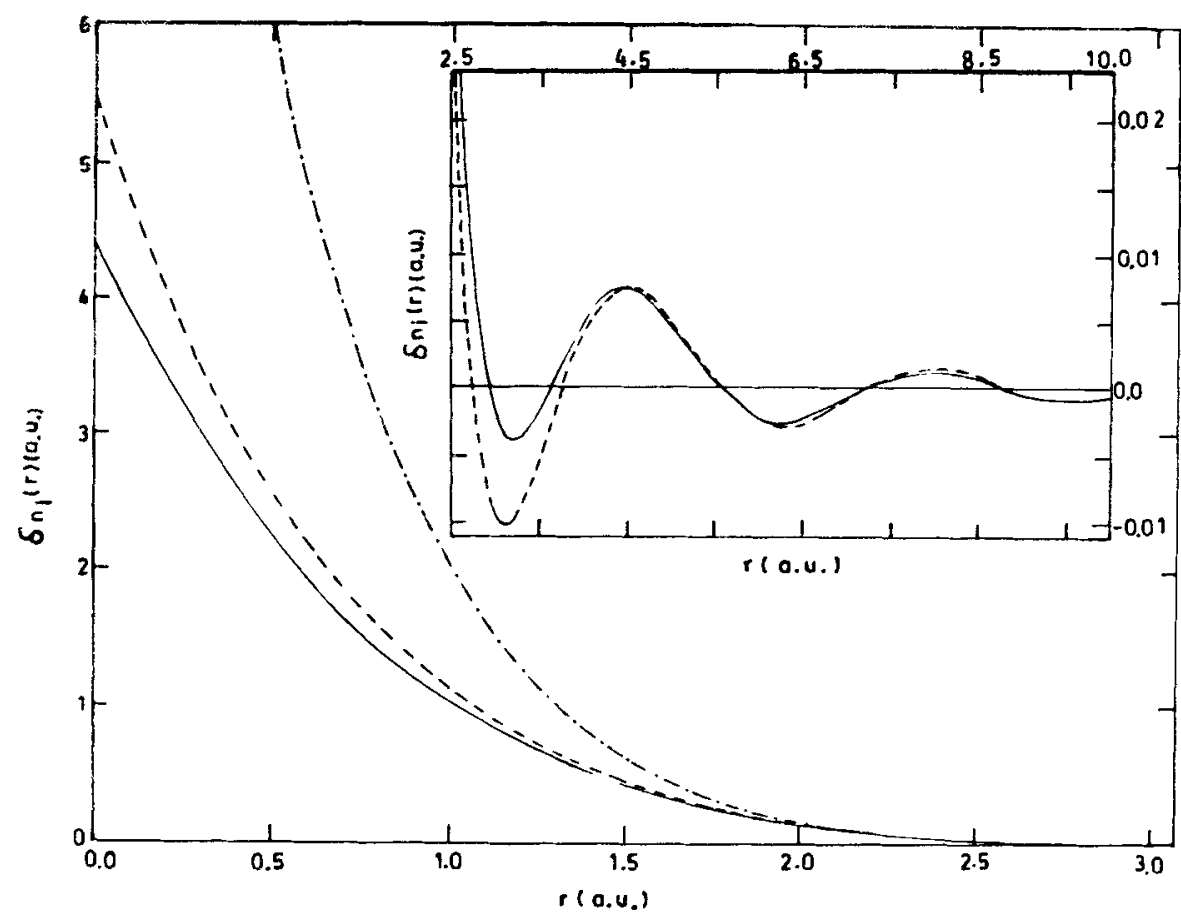

Figure 3. The ratio of proton induced charge density and electron charge density $\delta n_{1}(r) / n_{0}$. The rest of the description is the same as that of figure 2 .

$\mathrm{cm} / \mathrm{at} . \%$. Thus the lattice contribution enhances the residual resistivity by $2 \%$. On the other hand, the size effect reduces the residual resistivity by $58 \%$. Thus both the size effect and lattice contribution are required to give the correct description of residual resistivity of dilute metal-hydrogen system.

\section{Conclusion}

In this paper induced charge density is calculated by including the size effect and lattice contribution. The lattice contribution is included through spherical solid model. The Fermi surface is assumed to be essentially spherical. The discrete lattice and the relaxation effects are found to be essential to describe properly the effective charge, charge transfer and self-energy of the hydrogen impurity. A very shallow bound state of energy $-0.7450 \times 10^{-8}$ ryd. obtained using the present model suggests that there is no aluminium-hydride formation, i.e. proton exists at interstitial sites in $\mathrm{Al}$ matrix as free ions.

\section{Acknowledgements}

The authors wish to thank Prof. G K Mehta and Dr D K Avasthi for helpful discussions and suggestions. The authors are grateful to Prof. V C Sahni and Dr J K Sharma for encouraging us to carry out this work. The numerical calculations have been carried out with the PC/AT 386 purchased out of DST grant. 


\section{References}

Almbladh C O and von Barth U 1976 Phys. Rev. B13 3307

Beal-Monod M T and Kohn W 1968 J. Phys. Chem. Soiids 291877

Blatt F J 1957 Phys. Rev. 108 285, 1204

Flynn C P and Stoneham A M 1970 Phys. Ret. B1 3966

Friedel J 1952 Philos. Mag. 43153

Gunnarson O and Lundquist B I 1976 Phys. Rev. B13 4274

Jena P and Singwi K S 1978 Phy's. Ren. B17 3518

Kahn L M, Perrot F and Rasolt M 1980 Phys. Rev. B21 5594

Kohn W and Sham L J 1965 Phys. Rev. A140 1133

Manninen M and Nieminen R M 1979 J. Phys. F9 1333

Manninen M, Nieminen R. Hautojärvi P and Arponen J 1975 Phys. Rev. B12 4012

Perrot F and Rasolt M 1981 Phys. Rev. $\mathbf{B 3 3} 6534$

Popovic Z D, Stott M J, Carbotte J P and Piercy G R 1976 Phys. Rev. B13 590

Sholl C A and Smith P V 1978 J. Phys. F8 775 